the Boltzmann-Maxwell distribution, they will be so distributed at any subsequent instant. But in the absence of collisions or encounters between the system, this distribution will not be unique. There exist other distributions equally well satisfying the condition of $p$ rmanence.

The case of two or more molecules of gas in the process of enc sunter can be deduced, as shown in my Report ( $\$ 30$ ), by taking each "system" to repres nt a pair of molecules, there being at every instant an indefinitely large number of such pairs having different coordinates and momenta. It now seems definiiely settled that if the molecules are rigid bodies, the only possible permanent distribution unaffected by encounters is the Boltzmann-Maxwell distribution combined with motions of pure translation and pure rotation of the mass of gas as a whole (Report, § 46).

Many writers have limited their investigations to the case where all the encounters are binary, and I see that Prof. Fitzgerald has emplasised this point in his recent letter. I do not think this is the right place to draw the line. I can see no difficulty in taking account of encounters between three, four, or more molecules, provided that such encounters are sufficiently numerous to have a law of distribution. Where the line must be drawn is indicated in my Report, \$ 30 (iii.). The molecules which act on one another in any one encounter must form an infinitesimal fraction of the whole mass of gas, or the gas must at any instant be divisible into an infinitely large number of independent molecules and groups of molecules, each molecule or group thus constituting for the time being a free system by itself. When the mo'ecules act on each otber at all distances, or it is impossible to divide them into small independent groups, the whole of our theory breaks down.

It is this limitation which, to my mind, precludes our apply. ing the Kinetic Theory in its present state, not only to molecules moving ahout in a continuous medium, such as the ether may be, but also to solids and, probably, liquid:

A good deal of confusion has, I fear, ari en with regard to what $I$ have called Maxwell's La $N$ of Partition of Kinetic Energy, i.e. the statement that "if the kinetic energy of a system be expressed as a sum of squares, the mean vaiues of these squares are equal," from the fact that the term "mean value" may be taken to represent "time average for a single system" and I fancy this may be the point Prof. Fitzgerald had in view when he a-ked if the conclusions would not apply to the Earth or a "finite number of particles moving about for an indefinitely long time." It cannot be too strongly emphasised that this interpretation of mean values involves assumptions which have hardly been sufficiently justified in any general class of cases, and which have been repeatedly proved to be invalid in simple case; (Report, $\$ \$ 9-12$ ). ${ }^{1}$ To give a simple (if not quite analogous) illustration: suppose that on drawing counters out of a bag, we were to obtain on the average an equal number of red, white, and green counters. We should not be justified in inferring that if we kept a single counter sufficiently long it would change colour and become in turn red, white, and green.

As an illustration of a case where the Boltzmann-Maxwell Law is inapplicable, consider a sphere of density $\sigma$ moving in a perfect liquid of density $\rho$. The kinetic energies of the sphere and liquid are in the ratio of $\sigma: \frac{1}{2} \rho$, as we know from hydrodynamical considerations, and the same is true for any number of spheres whose distances apart are very large com. pared with their radii. This is not a case where no transference of energy takes place between the sphere and liquid, and it is not therefore open to Prof. Fitzgerald's objection to the case I previously cited. Whenever the motion of the solid is varied, energy is transferred to or from the liquid.

If a gaseous ether will satisfy the requirements of physicists, then the Boltzmann-Maxwell Law is unduubtedly applicable to the ether. If not, the ether falls entirely beyond the scope of our investigation. The Kinetic Theory is obviously a theory framed to account for one class of physical phenomena only, viz. the thermal properties of gases. If any observed phenomena are not deducible from the results of the theory, it is to my mind sufficient to show that this is because the funda. mental assumptions of the theory are not satisfied, i.e. that the phenumena in question are not inconsistent with the Kinetic Theory.

Peterhouse, Cambridge, January 16.

G. H. BRYAN.

I See also Nature, January 10, p. 262.

No. I 3 I 8 , vOI. 517

\section{Boltzmann's Minimum Function.}

I SAID in my first letter on this subject that the condition A, on which, or its equivalent, the proof is based, could not apply to the reversed motion. As that assertion has been questioned, may I confirm it thus?

The initial distribution of $R$, the relative velocity, i.e. the number of pairs of spheres for which it has given direction, is arbitrary - condition A is fulfilled. Then, as proved, whatever the initial distribution, after collisions, the distribution of $R$ is uniform, i.e. all directions equally probable. Now reverse the velocities. If condition $A$ is fulfilled in the reversed motion, then after the reversed collisions the distribution of $R$ is uniform. It is equally certain that it must be the same as the initial dis. tribution.

If, therefore, condition $\mathbf{A}$ is fulfilled in the reverse motion as well as in the direct, that can only be because the distribution of $\mathrm{R}$ was uniform to begin with. But that means that $\mathrm{H}$ was minimum to begin with, and therefore $\frac{d \mathrm{H}}{d t}=0$ throughout.

Boltzmann's theorem can be applied to both motions only on condition that it $h$ ts no effect in either.

I New-square, Lincoln's Inn.

S. H. BURBURY.

\section{Electroscopes in Lecture.}

I SUPPOSE teachers still use gold-leaf electroscopes for their junior lectures ; certainly I have found nothing else so dead. beat, or so readily understood; and by projecting with a lens a shadow of the leave; on a square-foot translucent screen, the movements are perfeetly visible to a large audience by day. light. But the one objection to the instrument, when used for explaining the fundamental facts, say, of induction, is that it indicates similarly positive and negative potentials. Yesterday, however, my assistant, Mr. E. E. Robinson, ingeniously stood the metal-cased instrument on a cake of paraffin wax, and electrified its outside negatively. By this process the zero of deflection is changed; the leaves stand apart for zero potential, diverge more for positive, and collapse for negative. A zero shadow-pointer and rough scale may be readily used; and I propose now to mount a projection electroscope in a suitable slightly-charged Leyden jar, whose outer cuat can then be treated a; the usual earthed terminal of the instrument, whose case is connected to or formed by the inner coat of the jar. The insulated or variable terminal is conveniently arranged as an insulated sphere or other shaped body on the lecture table, not far from the small screen, attached by a long enough thin wire to the leaves - of which it is perhaps best to have only one movable.

January 25.

OLIVER J. LODGE.

\section{The Perseid Meteors.}

YOUR interesting reference (NATURE, January 24, p. 301 ) to Dr. Bredichin's investigation of the Perseid shower, induces nie to offer one or two remarks on the subject. In the paragraph alluded to, it is stated that the radiant was observed from July 22 to September 12, 1893; but this long duration cannot possibly refer to the same system as that which furnishes the abundant maximum on August IO. It is true there are radiants in Perseus in September, and the succeeding months of October, November, and December supply many others, but, after the end of the third week in August, nothing is seen of the real Perseids. I cannot say exactly on how many nights the display continue; active, but it is certainly visible from July 19 to August I8, and the diurnal motion of the radiant is about $I^{\circ}$ to the E.N.E.

There are really very few of the true Perseids seen after August 12, for the shower dies rapidly away after the maximum. On August 1 5, 1893, during a watch of three hours, I saw only one Pers id. On August 16, 1893 , during a watch of $4^{\frac{1}{2}}$ hours, I noted only four Perseids. I mention these facts to prove the extreme tenuity of the stream at the middle of August. But there are differences from year to year. An observer watching the heavens for a similar interval on the same dates in succeed. ing years will get a varied experience. Thus one year cannot be regarded as the criterion of all.

Some years ago I recorded a few swift streak-leaving meteors between August 19 and 24 , from a radiant at $78^{\circ}+56^{\circ}$ on the northern borders of Auriga, and supposed they might be late 